\title{
A new technique that prevents paravalvular leakage after aortic valve replacement using a rapid deployment valve system
}

\author{
Taira Yamamoto ${ }^{1}$, Daisuke Endo ${ }^{2}$, Hironobu Yamaoka ${ }^{3}$, Satoshi Matsushita ${ }^{1}$, Kan \\ Kajimoto $^{1}$, Tohru Asai ${ }^{1}$, and Atsushi Amano ${ }^{1}$ \\ ${ }^{1}$ Juntendo University \\ ${ }^{2}$ Juntendo University - Hongo Campus \\ ${ }^{3}$ Edogawa Hospital
}

January 11, 2021

\begin{abstract}
Background and aim of the study: We report our one-year single-center experience of a new technique of aortic valve replacement using a rapid-deployment valve (RD-AVR) to avoid postoperative complications. We also report the unexpected pitfalls and handling techniques that we have seen in past cases. Methods: We performed aortic valve replacement on 38 patients between May 2019 and April 2020. Their mean age was 74 years. The primary outcomes were in-hospital mortality and short-term results during a one-year follow-up period, while the secondary outcomes were related to prosthetic valve function, especially paravalvular leakage (PVL). We further analyzed the relationship between the new technique and its outcomes. Results: The mean operative time was $196 \mathrm{~min}$. There were no in-hospital deaths, and the mean duration of postoperative hospital stay was 11.8 days. Valvular measurements using 3-dimensional computed tomography (3D-CT) were larger and more accurate than those measured using ultrasonic echocardiography Postoperative RD-AVR prosthetic valve function was excellent. However, PVL occurred in 4 cases one week and one year postoperatively and regurgitation did not improve. A gap associated with PVL was identified below the right-noncoronary commissure. To prevent PVL, we additionally stitched this gap in the later 18 cases; there was no case of PVL and no new pacemaker implantation in these cases. Conclusions: PVL is more likely to occur if there is a gap below the R-N commissure, especially in cases with a large annulus; therefore, applying an additional stitch to the R-N commissure is extremely useful.
\end{abstract}

\section{INTRODUCTION}

Degenerative calcific valve aortic stenosis (AS) caused by atherosclerosis is an active process of calcification and ossification and is the most common form of AS. ${ }^{1}$ Calcified AS prevalence is age-dependent, and aortic stenosis prevalence is reportedly $3 \%$ in people above the age of 75 years. ${ }^{2}$ Severe AS reportedly has a poor prognosis if untreated, even in the absence of symptoms. Although many doctors are aware of this fact, they are unable to persuade patients to undergo surgery because many patients often refuse it owing to the fear of surgical risks . Due to the widespread use of transcatheter aortic valve replacement (TAVR), cardiac surgeons are accumulating clinical data to prove the long-term prognosis and superior remote durability of aortic valve surgery.

Our fundamental goal was to prove the minimal invasiveness of aortic valve surgery by applying rapid deployment aortic valve replacement (RD-AVR) to new technologies and small incision approaches. The INTUITY Elite valve (Edwards Life Sciences, Irvine, CA, USA) is designed for rapid, easy, reproducible, and safe transplantation, with reduced operative time and surgical complications. ${ }^{3-5}$ However, studies reporting this prosthetic valve have vague cases and recommendations. ${ }^{6,7}$ The complications of this prosthetic valve include prosthetic valve regurgitation and arrhythmias, for example, in the complete atrioventricular block or left 
bundle branch block. We rarely have patients who experience hemolytic anemias and heart failure due to prosthetic valve regurgitation. In particular, paravalvular regurgitation (PVL) is a rare complication with a frequency of approximately $2.7 \%-17.8 \%$ (Figure 1); furthermore, we have found that perivalvular regurgitation can be predicted by electrocardiography-gated 3D-CT and can be resolved by a simple procedure. We present an implantation technique for aortic valve replacement along with collected data on optimal annular size and shape. Furthermore, we report our one-year single-center experience of 38 RD-AVR cases along with data on postoperative outcomes, valve-related paravalvular leakage, and hemodynamic performance.

\section{METHODS}

Patients:

We performed RD-AVR on 38 patients (mean age: $74.2 \pm 7.2$ years; range: 49-84 years) between April 2019 and March 2020.

\section{Interventional Technique:}

As in conventional surgical aortic valve replacement (SAVR), we placed patients on cardiopulmonary bypass, and the native leaflets were excised during cardiac arrest. The INTUITY Elite valve is designed to be compatible with conventional SAVR techniques with standard access to the aorta. In the first 20 patients in this series, we did not perform additional stitching, and PVL occurred in 4 patients. Therefore, in the latter 18 patients, a new stitch was added whenever there was a gap in the commissure.

We always performed debridement according to the traditional method of conventional SAVR. We believe that debriding all calcium from the annulus, left ventricular outflow tract (LVOT), anterior mitral valve leaflet, and sinus of the Valsalva is indispensable to ensure proper prosthetic valve positioning on the annulus and for optimal frame expansion.

After excising the calcified aortic valve and decalcifying the annulus, we must decide whether to use the INTUITY Elite valve because it is necessary to assess the interaction of the prosthesis with the aortic annulus. Failure to consider these factors can lead to implant failure and complications, such as PVL.

\section{INTUITY Elite valve (Edwards Life Sciences, Irvine, CA, USA)}

This system can be used in both conventional and minimally invasive surgeries, and it is covered by insurance even for patients on dialysis and for whom TAVR is not covered by insurance in Japan. The INTUITY Elite valve is designed to be compatible with conventional SAVR. However, the size of the delivery system is usually larger than the size of the prosthetic valve; hence, it may not pass through the sinotubular junction, and surgeons may have to choose smaller valves, which could increase the risk of paravalvular leakage. Conversely, bundle branch block or complete atrioventricular blocks can be induced by any resistance caused by the passage of the prosthetic valve through the annulus. Selecting the appropriate valve size is a critical step in the implantation of the INTUITY elite valve. How the valve sits on the annulus and how the inflow frame expands sub-annularly should be considered when selecting an appropriate sized valve.

We always used both the supra-and subannular sizing procedures. We used Magna Ease sizers to define the right valve diameter. We always avoided forcefully passing the valve through the annulus. Furthermore, we selected a size that we felt could effortlessly pass through the aortic annulus. If the measurement of the annulus is between two valve sizes, we always choose the smaller-sized valve.

If we choose a smaller sized valve, we may find a gap in the commissure (Figure 2A). It is essential to check for this gap to determine the location of the inserted prosthetic valve in the commissure. From our experience thus far, we have found that a gap more frequently occurs in the right coronary cusp (RCC)-noncoronary cusp (NCC) commissure than in the left coronary cusp (LCC)-RCC commissure. Even when aligned with the nadir, the prosthetic valve is located at the supra-annular position as the height of top of the commissure is greater than the INTUITY prosthetic valve's height; however, the valve is still in the infra-annular position at the commissure. 


\section{Suture place and Valve inspection}

After sizing the valve, we passed three evenly spaced sutures through the lowest point of each valvular cusp and the prosthetic valve to secure the prosthetic valve in the nadir of the annulus.

We always used non-pledgeted braided polyester-coated sutures with 2-0 non-cutting needles to place the three guiding sutures in the mid-commissural nadir region of the annulus. Using pledgets may create channels that result in PVL and could reduce the effective orifice area.

We inserted the suture from the lower end of the annulus and extracted the inserted needle from the Valsalva wall $4 \mathrm{~mm}$ above the annulus.

In cases with a gap in the commissure, we placed an additional everted mattress suture using 4-0 polypropylene monofilament sutures (Figure 2B,C). The position of the valve that we placed was $1 \mathrm{~mm}$ higher than the usual recommended position to avoid postoperative arrhythmias such as complete atrioventricular block or left bundle branch block. Again, we did not use pledgets.

We inspected carefully before closing the aortomy. The frame must be checked to ensure that it fits correctly into the distal LVOT. We believe that our additional sutures are an effective way to avoid situations in which there is a gap between the tissue under the annulus and the prosthetic valve in the commissure .

The gap in the smaller size-mismatched patient group was corrected entirely with the additional stitch before positioning the prosthetic valve (Figure $2 \mathrm{~B}$ ).

\section{Data collection:}

All 38 consecutive patients underwent successful isolated or combined RD-AVR between May 2019 and April 2020 in the Department of Cardiac Surgery at the Juntendo University Hospital. The institutional review board approved this registry, and patients signed informed consent preoperatively. The comprehensive safety and effectiveness of RD-AVR were assessed. These data included preoperative native valve function, postoperative structural/non-structural valve dysfunction, and hemodynamic valve performance on TTE and electrocardiography-gated 3D-CT.

The primary outcome was hospital mortality and short-term results after a one-year follow-up. Secondary outcomes relating to prosthetic valve function, especially PVL, were analyzed. Subset analyses were performed to determine the relationship between new technical methods and outcomes.

\section{Statistical Analysis:}

We assess the normality of the distribution of continuous variables using D'Agostino-Pearson analysis, we used descriptive statistical methods to depict the study population and presented continuous variables as means and standard deviations. We also reported the total number and proportions of categorical outcomes. We showed results as early (one year later) events and noted possibilities as numbers and percentages. We compared continuous variables using the t-test or Wilcoxon or Mann-Whitney U tests (non-parametric variables) and made comparisons of categorical variables using the Pearson's v2 test. A two-tailed p-value of $<0.05$ was considered significant. We analyzed all data with JMP 14.0 software (SAS Institute, Cary, NC).

\section{RESULTS}

\section{Demographics and baseline characteristics}

Between January 2020 and March 2021, we performed SAVR using the INTUITY Valve System for 38 AS patients at our institution. The mean age of the patients was 74.2 (SD 7.2) years (range 49-84 years), and $10(26.3 \%)$ patients were female. Most patients had a history of hypertension (73.7\%) and hyperlipidemia $(78.9 \%)$. Coronary artery disease, diabetes, and cerebrovascular accidents were present in $36.8 \%, 34.2 \%$, and $15.8 \%$ of patients, respectively. We estimated the predicted operative risk preoperatively through the European System for Cardiac Operative Risk Evaluation II (EuroSCORE II) and the Society of Thoracic 
Surgeons scores (STS scores), which showed early mortalities of 2.79 (SD 2.63) and 4.92 (SD 3.85), respectively. Patient baseline characteristics and cardiovascular and systemic comorbidities are listed in Table 1.

The preoperative mean pressure gradient (PG) over the aortic valve was 40 (SD 15.6) $\mathrm{mmHg}$, and the preoperative mean aortic valve area was $0.8(\mathrm{SD} 0.2) \mathrm{cm}^{2}$. Table 2 shows an overview of the preoperative echocardiographic findings of patients.

We performed RD-AVR via median sternotomies in 28 (65.8\%) patients and via upper-hemisternotomies in $13(34.2 \%)$ patients, and there was no case of conversion to other procedures. Concomitant procedures were performed in 13 patients $(34.2 \%)$. We also performed coronary artery bypass grafting (CABG) in $9(23.7 \%)$ patients; other procedures are detailed in Table 2. Cardiopulmonary bypass (CPB) and cross-clamp times were 196 (SD, 71) and 65 (SD, 24) min, respectively (Table 3).

\section{Clinical outcomes}

No in-hospital mortality occurred among the patients in this study. No neurological events, low output syndrome, respiratory complications, or renal failure occurred. We had only one case of an early bleeding event that needed revision; however, no late major bleeding events occurred. New pacemaker implantation was required in only one patient (2.6\%) during the first 30 postoperative days (Table 3 ).

The most common size implanted was $23 \mathrm{~mm}$ (42\%), followed by $25 \mathrm{~mm} \mathrm{(26 \% ),} 21 \mathrm{~mm} \mathrm{(21 \% ),} \mathrm{and} 19 \mathrm{~mm}$ (11\%). We evaluated patient preoperative baseline values for each valve. An increased proportion of bicuspid valves was associated with large valves, the body surface area of such valves, and male gender. At sizes of $19 \mathrm{~mm}$ and $21 \mathrm{~mm}$, valve sizes were almost the same as the measured value on echocardiography, but in cases where the artificial valve used was large, sizes were underestimated, and a difference occurred. In such cases, the diameter of the sinotubular junction is expanded, and the overall image of aortic root showed that the root is dilated. The value measured by $3 \mathrm{D}-\mathrm{CT}$ was larger and more accurate than that by ultrasonic echocardiography(UCG). (Table 4)

We reported the follow-up clinical outcomes for each implanted valve size (Table 5). The mean gradients, effective orifice area, and effective orifice area index at the 1-year follow-up were excellent in all cases. No prosthesis-patient mismatch (PPM) was observed during follow-up. However, postoperative PVL was found in four patients (10.5\%); of these patients, three PVLs were only mild, and only one was moderate.

The PVL did not disappear even after a year. All four cases with regurgitation were large in prosthetic valve size, and one patient had a type 1 bicuspid aortic valve. One patient had a small gap and had hemolytic anemia; therefore, he underwent reoperation one year later (Figure 3). A case of regurgitation around the prosthetic valve was examined using 3D-CT. The valve-annulus gap was associated with a larger annulus, regardless of whether it was a perfect circle or an ellipse (Table 6).

There were no complications or PVL and no complete AV block during hospitalization because we performed the aforementioned extra stitch (Table 7). Consequently, almost all patients were discharged without any complications.

\section{CONCLUSIONS}

A partner cohort study [8] showed that PVL (more than mild) was associated with an increase in the incidence of late mortality and severe PVL in the Pivotal Extreme Risk Trial. ${ }^{9}$ A meta-analysis by Athappan et al. ${ }^{10}$ showed that in patients with moderate and severe PVL, the 1-year survival rate was reduced.

The presence of mild or more significant PVL after TAVR increases the incidence of mortality in the longterm. ${ }^{11,12}$ In the REPRISE III trial, the incidence of stroke events was increased in patients with mild PVL after TAVR. This indicated an association between PVL and stroke events. This raises the question of whether PVL may increase the risks of other postoperative complications. ${ }^{13}$ No practical method or uniformly used strategy for predicting PVL has been reported yet. It has been shown that one of the most significant predictors of paravalvular aortic regurgitation are valvular and annular calcium, which can 
predict PVL with a sensitivity and specificity of $86 \%$ and $80 \%$, respectively, if the valvular calcium score was above 3,000. ${ }^{14}$ By applying such information, the sensitivity and specificity of the presence of cyclic calcium (i.e., asymmetric calcium) is a moderate predictor of Grade I or higher PVL. ${ }^{15}$ Reiff et al. reported that reproducing a high-fidelity model of the human aortic root was feasible in a clinical setting and did not require composite models and expensive printers. Furthermore, implanting a TAVR valve within a 3D printed model could aid predicting PVL. In the absence of adequate therapy for PVL, it is essential to develop a high-fidelity computational model to predict PVL. ${ }^{15}$ Revolutionary valve-implantation technology and improved preoperative planning with electrocardiography-gated multislice 3D-CT have overcome some complex problems ${ }^{16}$; however, PVL remains a problem in TAVR. ${ }^{17}$ There is minimal PVL in conventional SAVR because the principle of SAVR is to remove as much calcification as possible in and around the annulus during SAVR. Many reports suggest that PVL does not affect mortality, cardiac accidents, deterioration of prosthetic valves, or artificial valve infection in the long-term. ${ }^{18,19}$ However, in addition to the above information, a meta-analysis of TAVR raised concerns that even mild perivalvular regurgitation can be detrimental to patient survival. ${ }^{20,21}$

RD-AVR has a low rate of PVL (severe aortic regurgitation(AR) 0.2\%, moderate AR 1.2\%, mild AR 6.1\%), but these values are still higher than those reported after conventional SAVR interventions. ${ }^{22}$

The rate of significant postoperative PVL was $3.7 \%$ in the bicuspid aortic valve. These data are similar to those reported in previous studies. However, the risk of postoperative AR is 2.5 times higher than that of the total population of the SURD registry. We believe that it is essential to understand the complexity of the anatomy of bicuspid aortic valve (BAV) and restore the annular circumference for complete results. ${ }^{6,} 22$

To reduce PVL incidence, Andreas et al. ${ }^{23}$ reported the application ofan extra stitch in the noncoronary sinus in selected patients, and they did not implant this particular type of valve in patients with extensive calcifications of the root with rigid sinuses. Furthermore, SURD-IR data collection for more than 12 years showed that the incidence (17.8\% to $2.7 \%$ ) (Figure 1)and severity (severe AR $0.6 \%$ to 0, moderate AR $3.1 \%$ to $1.1 \%$, and mild AR. $14.1 \%$ to $1.6 \%$ ) of postoperative PVL decreased significantly. This may be due to the increased surgical experience in RD-AVR surgery. ${ }^{24}$

By observing preoperative data, Chirichilli et al. found that bicuspid aortic valves have a larger mean annular size in terms of area and perimeter compared to tricuspid aortic valves. This preprocedural anatomical finding might explain why preoperative CT scans showed an elliptic shape of TAVs (Ellipticity Index 1.3 +- 0.1), a circular shape of type 0 BAVs $(1.1+-0.1)$ and an intermediate behavior of type 1 BAVs; this suggested a possible gradual spectrum of TAV circularity from type 1 BAV to type 0 BAV circularities. ${ }^{25}$ In our study, the 3D-CT reading was larger than the UCG reading, and the actual replacement prosthesis size was closer to 3D-CT tasks. Preoperative ellipse was not associated with perivalvular regurgitation, but perivalvular regurgitation was more common in patients with a large aortic annulus. This may be related to the large aortic annulus and the possible loss of active elasticity in the bicuspid valve.

We encountered four cases of PVL in the first half of this series, and one patient had hemolytic anemia, which required the replacement of the aortic valve. In our experience, regurgitation from the RCC-NCC commissure was the most common form, followed by regurgitation from the LCC-RCC commissure. It has been found that choosing a smaller prosthesis to avoid implantation of a new pacemaker or left bundle branch block increases the likelihood of having a gap in the commissure. If the height of the prostheticvalve is adjusted to the nadir, it will be in the infra-annular position at the commissure. Therefore, it was also found that a form with the commissure being high and spreading outwards had much regurgitation around the prosthetic valve. This morphology is especially common in bicuspid aortic valves. Ferrari et al. reported that the aortic annulus stabilization technique based on an already performed annuloplasty of the aortic annulus that uses a running 3-0 polypropylene suture was sufficient to prevent PVL. ${ }^{7}$ However, our findings suggest that the elliptical shape of the annulus is not associated with PVL, and this is owing to a gap under the RCC-NCC commissure. Therefore, the additional stitch that we performed was easy and quick.

We changed the technique in the last 18 cases and inserted an everted mattress stitch at the commissure; the 
artificial valve was pulled up to the commissure and fixed. This extra stitch eliminated PVL complications, and there were no PVL incidences in the later 18 cases. We often choose a smaller-sized valve RD-AVR to avoid complete atrioventricular and left bundle branch blocks. In such cases, we are concerned about PVL, but we think this new stitch is a very effective way of eliminating PVL.

This study has some limitations. This was a retrospective case series with a small number of patients, meaning that there is also a risk of bias; there was also a potential for selection bias given that participants were all from a single university hospital. This did not allow us generalize the results of this study to different populations. Additionally, we reported only RD-AVR cases and did not show comparative data with conventional SAVR and TAVR. In future studies, we need to confirm the anatomical analysis of PVL for all aortic valve stenosis patients and long-term results and compare patients with and without PVL.

PVL is a rare complication of RD-AVR that may require reoperation and should be avoided. 3D-CT can measure PVL more accurately than UCG and is a useful test method for predicting PVL. We conclude that PVL is more likely to occur if there is a gap below the R-N commissure, especially in cases with large annulus sizes. In such cases, applying an additional stitch to the R-N commissure is extremely useful.

Acknowledgments: We would like to thank Editage (www.editage.com) for English language editing.

\section{Authors' contributions:}

TY and DE were the primary operators during RD-AVR, and collected and analyzed clinical data. TY and DE contributed equally to the writing of the manuscript. HY analyzed the results of surgical methods when combined together. SM, KK, and AS conducted the operative management of patients at the Juntendo University and collected papers regarding RD-AVR. AA supervised the work. All authors read and approved the final manuscript.

\section{REFERENCES}

1. Thaden JJ, Nkomo VT, Enriquez-Sarano M. The global burden of aortic stenosis. Prog Cardiovasc Dis 2014:56;565-571.

2. Nkomo VT, Gardin JM, Skelton TN, Gottdiener JS, Scott CG, Enriquez-Sarano M. Burden of valvular heart diseases: a population-based study. Lancet 2006;368:1005-1011.

3. Glauber M, Miceli A. Minimally invasive aortic valve replacement with sutureless valve is the appropriate treatment option for high-risk patients and the "real alternative" to transcatheter aortic valve implantation. J Thorac Cardiovasc Surg 2016;151:610-613.

4. Coti I, Haberl T, Scherzer S, et al. Outcome of rapid deployment aortic valves: long-term experience after 700 implants. Ann Cardiothorac Surg 2020;9:314-321.

5. Sadri V, Bloodworth CH 4th, Madukauwa-David ID, Midha PA, Raghav V, Yoganathan AP. A mechanistic investigation of the EDWARDS INTUITY Elite valve's hemodynamic performance. Gen Thorac Cardiovasc Surg 2020;68:9-17

6. Miceli A, Berretta P, Fiore A, et al. Sutureless and rapid deployment implantation in bicuspid aortic valve: results from the sutureless and rapid-deployment aortic valve replacement international registry. Ann Cardiothorac Surg 2020;9:298-304.

7. Ferrari E, Siniscalchi G, Tozzi P, von Segesser L. Aortic annulus stabilization technique for rapid deployment of aortic valve replacement. Innovations (Phila). 2015;10:360-362.

8. Kodali SK, Williams MR, Smith CR, et al. Two-year outcomes after transcatheter or surgical aorticvalve replacement N Engl J Med 2012;366:1686-1695.

9. Popma JJ, Adams DH, Reardon MJ, et al. Transcatheter aortic valve replacement using a selfexpanding bioprosthesis in patients with severe aortic stenosis at an extreme risk of surgery. J Am Coll Cardiol 2014;63:1972-1981.

10. Athappan G, Patvardhan E, Tuzcu EM, et al. Incidence, predictors, and outcomes of aortic regurgitation after transcatheter aortic valve replacement: A meta-analysis and systematic review of literature. J AmColl Cardiol 2013;61:1585-1595. 
11. Thourani VH, Kodali S, Makkar RR, et al. Transcatheter aortic valve replacement versus surgical valve replacement in intermediate-risk patients: A propensity score analysis. Lancet 2016;387:2218-2225.

12. Hayashida K, Lefevre T, Chevalier B, et al. Impact of post-procedural aortic regurgitation on mortality after transcatheter aortic valve implantation JACC Cardiovasc Interv 2012;5:1247-1256.

13. Feldman TE, Reardon MJ, Rajagopal V, et al. Effect of mechanically expanded vs. self-expanding transcatheter aortic valve replacement on mortality and major adverse clinical events in high-risk patients with aortic stenosis: the REPRISE III randomized clinical trial. J Am Med Assoc 2018;319:2737.

14. Colli A, Gallo M, Bernabeu E, et al. Aortic valve calcium scoring is a predictor of paravalvular aortic regurgitation after transcatheter aortic valve implantation. Ann Cardiothor Surg 2012;1:156-159.

15. Reiff C, Zhingre Sanchez JD, Mattison LM, et al. 3-Dimensional printing to predict paravalvular regurgitation after transcatheter aortic valve replacement. Catheter Cardiovasc Interv 2020;96:E703E710.

16. Van Belle E, Juthier F, Susen S, et al. France 2 Investigators. Postprocedural aortic regurgitation in balloon-expandable and self-expandable transcatheter aortic valve replacement procedures: analysis of predictors and impact on long-term mortality: insights from the FRANCE2 Registry. Circulation 2014;129:1415-1427.

17. Feldman TE, Reardon MJ, Rajagopal V, et al. Effect of mechanically expanded vs. self-expanding transcatheter aortic valve replacement on mortality and major adverse clinical events in high-risk patients with aortic stenosis: the REPRISE III randomized clinical trial. J Am Med Assoc 2018;319:2737.

18. Ionescu A, Fraser AG, Butchart EG. Prevalence and clinical significance of incidental paraprosthetic valvar regurgitation: a prospective study using transesophageal echocardiography. Heart 2003;89:13161321.

19. Rallidis LS, Moyssakis IE, Ikonomidis I, et al. Natural history of early aortic paraprosthetic regurgitation: A five-year follow-up. Am Heart J 1999;138:351-357.

20. Ando T, Briasoulis A, Telila T, et al. Does mild paravalvular regurgitation post transcatheter aortic valve implantation affect survival? A meta-analysis. Catheter Cardiovasc Interv 2018; 91:135-147.

21. Flynn CD, Williams ML, Chakos A, Hirst L, Muston B, Tian DH. Sutureless valve and rapid deployment valves: A systematic review and meta-analysis of comparative studies. Ann Cardiothorac Surg 2020:9;364-374.

22. Di Eusanio M, Phan K, Berretta P, et al. Sutureless and Rapid-Deployment Aortic Valve Replacement International Registry (SURD-IR): Early results from 3343 patients. Eur J Cardiothorac Surg 2018;54:768-773.

23. Andreas M, Coti I, Rosenhek R, et al. Intermediate-term outcome of 500 consecutive rapid-deployment surgical aortic valve procedures. Eur J Cardiothorac Surg $2019 ; 55: 527-533$.

24. Di Eusanio M, Berretta P. The sutureless and rapid-deployment aortic valve replacement international registry: lessons learned from more than 4,500 patients. Ann Cardiothorac Surg. 2020;9:289-297.

25. Chirichilli I, Irace F, Weltert L, et al. Morphological modification of the aortic annulus in tricuspid and bicuspid valves after aortic valve reimplantation: An electrocardiography-gated computed tomography study Eur J Cardiothorac Surg 2019;56:778-784.

\section{TABLES}

\section{Table 1 Preoperative baseline}

\begin{tabular}{ll}
\hline Age $($ year $)$ & $74.2+7.2(49-84)$ \\
\hline Female gender & $10(26.3 \%)$ \\
Body mass index $\left(\mathrm{kg} / \mathrm{m}^{2}\right)$ & $23.59+3.50$ \\
Body surface area $\left(\mathrm{m}^{2}\right)$ & $1.63+0.15$ \\
Euro Score II & $2.79+2.63$
\end{tabular}




\begin{tabular}{ll}
\hline Age (year) & $74.2+7.2(49-84)$ \\
\hline STS Score & $4.92+3.85$ \\
Brain natriuretic peptide (pg/mL) & $325+589$ \\
Diabetes Mellitus & $13(34.2 \%)$ \\
Hypertension & $30(78.9 \%)$ \\
Hyperlipidemia & $28(73.7 \%)$ \\
Coronary artery disease & $14(36.8 \%)$ \\
Previously PCI & $6(16.7 \%)$ \\
Cerebrovascular accident & $6(15.8 \%)$ \\
Estimated glomerular filtration (ratemL/min/1.73 m2) & $48.2+29.2$ \\
Hemodialysis & $10(26.3 \%)$ \\
Peripheral vascular disease & $2(5.2 \%)$ \\
Chronic lung disease & $5(13.2 \%)$ \\
Malignancy & $6(15.7 \%)$ \\
Hemoglobin(mg/dl) & $12.6+1.8$ \\
Albumin(mg/dl) & $3.9+0.5$ \\
Previous cardiovascular interventions & $6(15.8 \%)$ \\
Previous cardiac surgery & $1(2.6 \%)$ \\
Previous pacemaker implantation & 0 \\
Previous rhythm abnormalities & $2(5.2 \%)$ \\
Previous atrial fibrillation & $3(7.9 \%)$ \\
\hline
\end{tabular}

EuroSCORE: European system for cardiac operative risk evaluation

Table 2 Preoperative ultrasonic echocardiography

\begin{tabular}{ll}
\hline Left ventricular ejection fraction $(\%)$ & $65+8$ \\
\hline Valsalva $(\mathrm{mm})$ & $32.6+3.7$ \\
Sinotubular junction $(\mathrm{mm})$ & $26.1+3.6$ \\
Annulus $(\mathrm{mm})$ & $21.4+2.5$ \\
Aortic valve area $\left(\mathrm{cm}^{2}\right)$ & $0.8+0.2$ \\
Mean pressure gradient $(\mathrm{mmHg})$ & $40.1+15.6$ \\
Velocity $(\mathrm{m} / \mathrm{s})$ & $4.2+0.8$ \\
\hline
\end{tabular}

Table 3 Intraoperative patient characteristics and early results

\begin{tabular}{ll}
\hline Full sternotomy access & $25(65.8 \%)$ \\
\hline Hemi-sternotomy access & $13(34.2 \%)$ \\
Concomitant procedures & \\
CABG & $9(23.7 \%)$ \\
Aortic surgery (total arch replacement) & $2(5.3 \%)$ \\
Mitral valve plasty & $1(2.6 \%)$ \\
Atrial fibrillation surgery & $4(10.5 \%)$ \\
Left atrial appendage amputation & $38(100 \%)$ \\
Morrow procedure & $1(2.6 \%)$ \\
Implanted valve size (mm) & \\
19 & $4(10.5 \%)$ \\
21 & $8(21.1 \%)$ \\
23 & $16(42.1 \%)$
\end{tabular}




\begin{tabular}{ll}
\hline Full sternotomy access & $25(65.8 \%)$ \\
\hline 25 & $10(26.3 \%)$ \\
Operative time (min) & $196+71$ \\
Cross-clamp time (min) & $65+24$ \\
Cardiopulmonary bypass time (min) & $95+41$ \\
Hospital mortality & 0 \\
Low output syndrome & 0 \\
Cerebrovascular accident & 0 \\
Respiratory trouble & 0 \\
Infection & 0 \\
Renal failure & 0 \\
Revision for bleeding & $1(2.6 \%)$ \\
New pacemaker implantation & $1(2.6 \%)$ \\
New onset of atrial fibrillation & $11(28.9 \%)$ \\
ICU stay (hours) & $31+20$ \\
Hospital stay (days) & $11.8+6.3$ \\
\hline
\end{tabular}

CABG: coronary artery bypass grafting

ICU: Intensive Care Unit

Table 4 Preoperative baseline characteristics among each implanted RD-AVR valve

\begin{tabular}{|c|c|c|c|c|}
\hline Size & $19 \mathrm{~mm}$ & $21 \mathrm{~mm}$ & $23 \mathrm{~mm}$ & $25 \mathrm{~mm}$ \\
\hline No of cases & 4 & 8 & 16 & 10 \\
\hline Age (years old) & $73+3$ & $75+3$ & $75+5$ & $72+11$ \\
\hline Female gender & $4(100 \%)$ & $6(75 \%)$ & 0 & 0 \\
\hline BMI (kg/m2 & $24.9+3.1$ & $23.4+5.5$ & $23.2+3.4$ & $23.8+3.1$ \\
\hline $\mathrm{BSA}(\mathrm{m} 2)$ & $1.49+0.07$ & $1.51+0.11$ & $1.67+0.13$ & $1.74+0.15$ \\
\hline $\begin{array}{l}\text { Bicuspid aortic } \\
\text { valve }\end{array}$ & 0 & 0 & $3(18.8 \%)$ & $5(50 \%)$ \\
\hline $\begin{array}{l}\text { Annulus (mm) } \\
\text { UCG }\end{array}$ & $18.7+0.5$ & $20.5+2.6$ & $21.2+2.0$ & $23.9+1.9$ \\
\hline $\begin{array}{l}\text { Annulus (mm) } \\
\text { 3DCT }\end{array}$ & $20.1+1.8$ & $22.2+1.7$ & $22.5+6.3$ & $25.8+1.6$ \\
\hline $\begin{array}{l}\text { Valsalva }(\mathrm{mm}) \\
\text { UCG }\end{array}$ & $26.9+1.2$ & $30.1+2.3$ & $33.8+2.8$ & $35.2+3.3$ \\
\hline $\begin{array}{l}\text { Valsalva }(\mathrm{mm}) \\
\text { 3DCT }\end{array}$ & $29.0+2.2$ & $32.8+3.5$ & $34.2+2.3$ & $35.5+2.7$ \\
\hline STJ (mm) UCG & $21.7+1.6$ & $23.5+1.6$ & $26.8+3.6$ & $28.6+2.8$ \\
\hline STJ (mm) 3DCT & $24.4+2.7$ & $26.8+1.9$ & $28.0+2.1$ & $31.7+2.7$ \\
\hline $\begin{array}{l}\text { LVOT (mm) } \\
\text { UCG }\end{array}$ & $18.4+0.3$ & $20.6+1.5$ & $21.4+1.6$ & $23.1+2.1$ \\
\hline $\begin{array}{l}\text { LVOT (mm) } \\
3 \text { DCT }\end{array}$ & $20.6+1.5$ & $22.0+2.0$ & $23.0+1.0$ & $25.2+1.6$ \\
\hline $\operatorname{AVA}\left(\mathrm{m}^{2}\right)$ & $0.73+0.15$ & $0.81+0.23$ & $0.82+0.24$ & $0.98+0.24$ \\
\hline $\begin{array}{l}\text { Aortic regurgitation } \\
(>\text { moderate) }\end{array}$ & $1(25.0 \%)$ & $2(28.5 \%)$ & $4(26.7 \%)$ & $5(50.0 \%)$ \\
\hline
\end{tabular}

Table 5 Echocardiographic data 12 months postoperatively 


\begin{tabular}{lllll}
\hline Size & $19 \mathrm{~mm}$ & $21 \mathrm{~mm}$ & $23 \mathrm{~mm}$ & $25 \mathrm{~mm}$ \\
\hline Number of cases & 4 & 8 & 16 & 10 \\
Mean aortic gradient $(\mathrm{mmHg})$ & $13.4+6.4$ & $10.8+3.2$ & $9.5+3.2$ & $8.0+3.8$ \\
Effective orifice area $\left(\mathrm{cm}^{2}\right)$ & $1.41+0.14$ & $1.84+0.28$ & $2.02+0.16$ & $2.26+0.47$ \\
Effective orifice area index $\left(\mathrm{cm}^{2} / \mathrm{m}^{2}\right)$ & $0.97+0.10$ & $1.16+0.23$ & $1.24+0.16$ & $1.30+0.24$ \\
Paravalvular leakage & 0 & 0 & $2(12.5)$ & $2(20.0)$ \\
AR mild & 0 & 0 & 1 & 2 \\
AR moderate & 0 & 0 & 1 & 0 \\
New PM implantation & $1(25 \%)$ & 0 & 0 & 0 \\
New LBBB & 0 & 1 & 3 & 0 \\
\hline
\end{tabular}

AR: aortic regurgitation; LBBB: Left bundle branch block; PM: pacemaker

Table 6 Annular shape indicated by electrocardiography-gated 3-dimensional computed tomography

\begin{tabular}{llll}
\hline & PVL $(\mathrm{n}=4)$ & No PVL $(\mathrm{n}=34)$ & P-value \\
\hline Major axis of annuls (MD) $(\mathrm{mm})$ & $29.5+0.9$ & $26.1+2.3$ & 0.01 \\
Minor axis of annulus (mD) $(\mathrm{mm})$ & $23.5+2.3$ & $21.2+2.1$ & 0.04 \\
Ellipticity (PI = MD/mD) & $1.26+0.09$ & $1.23+0.08$ & 0.54 \\
Annulus circumference (mm) & $82.8+5.0$ & $73.2+6.7$ & 0.01 \\
Aortic valve annular area (m2) & $528+71$ & $404+99$ & 0.02 \\
\hline
\end{tabular}

Table 7 Durability, paravalvular leakage, and arrhythmia between the first 10 cases and the last 18 cases

\begin{tabular}{lll}
\hline & First 20 cases & Last 18 Cases \\
\hline Effective orifice area index $(\mathrm{cm} 2 / \mathrm{m} 2)$ & $1.20+0.19$ & $1.30+0.25$ \\
Paravalvular leakage & $4(20.0)$ & 0 \\
New PM implantation & 1 & 0 \\
New LBBB & 1 & 3 \\
\hline
\end{tabular}

LBBB: Left bundle branch block

Figure legends

Figure 1. Paravalvular leakage confirmed by transesophageal echocardiography

Figure 2. How to apply the additional stitch

2A: The ST-junction is smaller than annulus, and the gap is recognized below the commissure.

2B: The red arrow line is the line of sewing cuff, and the blue line is the commissure. Moreover, there was a gap in the vertical direction. The yellow arrows indicate additional stitch lines.

$2 \mathrm{C}$ : We applied the additional stitch, which is everted an horizontal mattress suture without pledgets.

Figure 3. Paravalvular leakage after one year of follow-up

A $2 \mathrm{~mm}$ sonde is passed through the gap between the artificial valve and the annulus. 


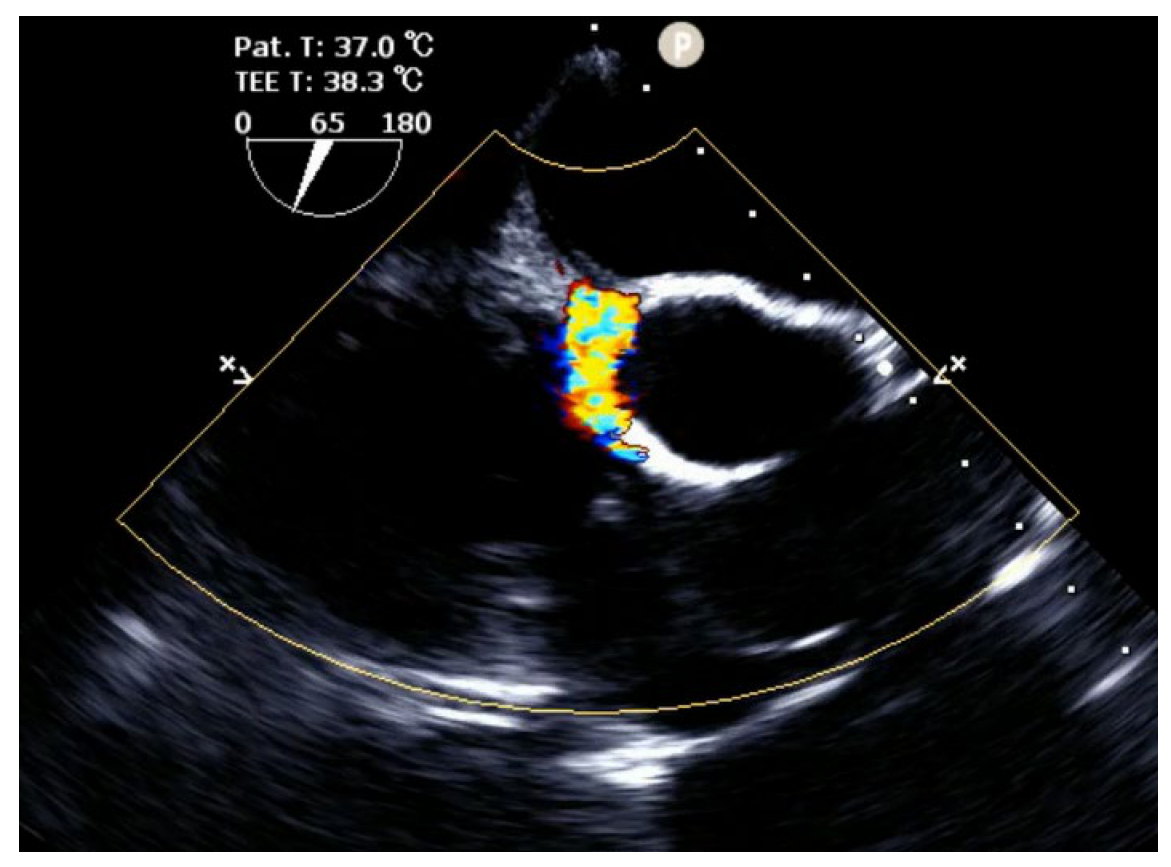

figures/Fig-2/Fig-2-eps-converted-to.pdf 


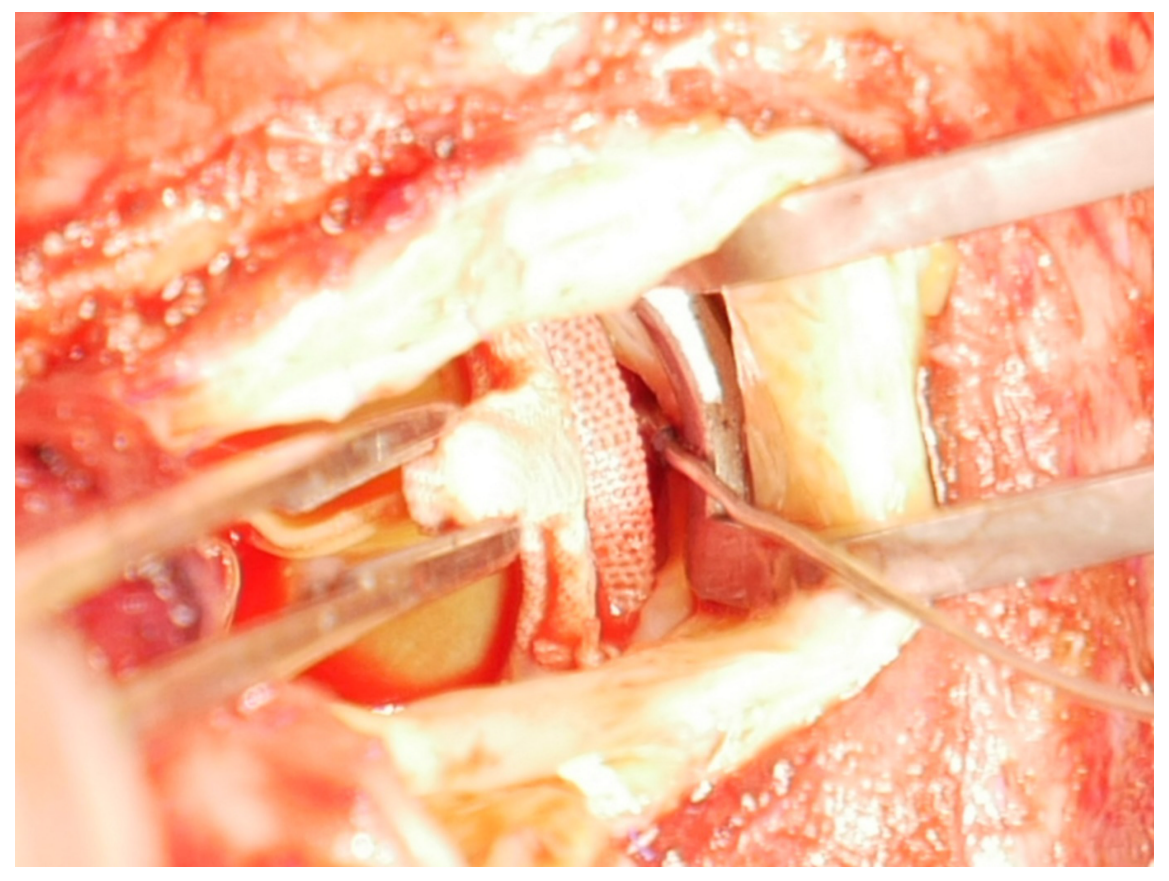

